\title{
Competition of information channels in the spreading of innovations
}

\author{
Gergely Kocsis and Ferenc Kun* \\ Department of Theoretical Physics, University of Debrecen, P.O. Box 5, H-4010 Debrecen, Hungary
}

(Received 7 June 2010; revised manuscript received 13 May 2011; published 15 August 2011)

\begin{abstract}
We study the spreading of information on technological developments in socioeconomic systems where the social contacts of agents are represented by a network of connections. In the model, agents get informed about the existence and advantages of new innovations through advertising activities of producers, which are then followed by an interagent information transfer. Computer simulations revealed that varying the strength of external driving and of interagent coupling, furthermore, the topology of social contacts, the model presents a complex behavior with interesting novel features: On the macrolevel the system exhibits logistic behavior typical for the diffusion of innovations. The time evolution can be described analytically by an integral equation that captures the nucleation and growth of clusters of informed agents. On the microlevel, small clusters are found to be compact with a crossover to fractal structures with increasing size. The distribution of cluster sizes has a power-law behavior with a crossover to a higher exponent when long-range social contacts are present in the system. Based on computer simulations we construct an approximate phase diagram of the model on a regular square lattice of agents.
\end{abstract}

DOI: 10.1103/PhysRevE.84.026111

PACS number(s): 05.65.+b, 87.23.Ge, 02.50.Le, 87.23.Kg

\section{INTRODUCTION}

Technological evolution of socioeconomic systems has two major components [1-4]: (1) Innovation-New products, ideas, and paradigms emerge as a result of innovations, which are then tested by the market. (2) Spreading-Successful technologies spread over the system, resulting in an overall technological progress. The understanding of the technological progress of socioeconomic systems requires the analysis of the complex dynamics of several levels from the interaction of firms investing in research and doing production [5-7], through the emergence of new ideas captured by the network of patents [8], down to the level of individuals that use the newly developed products [9-12]. A crucial element of the diffusion of technological advancements is the spreading of information about the existence and advantages of the newly developed technology. This process is usually initiated by the advertising activities of producers and providers of the new product, resulting in a vertical information flow into the system. Next, information is transmitted laterally through the communication of individuals.

The effect of word-of-mouth communication on the spreading of information in socioeconomic systems has recently been studied to explain the emergence of extreme market shares. This phenomenon means that in certain markets either hits or failures of newly introduced products appear $[13,14]$. It was shown that social percolation occurring due to information transmission through social contacts and to quality adjustment of producers can be responsible for extreme market shares. Such systems were found to evolve toward self-organized criticality in the vicinity of the percolation threshold [14]. The model of social percolation was further extended by considering the effect of mass media that acts as an external information source, however, only at the beginning of the spreading process [15]. Diffusion of innovations also involves

*feri@dtp.atomki.hu components analogous to opinion spreading and consensus formation [9-12,16-27]. As an example, telecommunication technologies can be mentioned, where the usage of a technology assumes that the communication partners are approximately at the same technological level $[1,9-12,19,20]$.

In the present paper we focus on the spreading of information in socioeconomic systems about the availability and advantages of new products investigating the competition of the vertical and horizontal information channels. The system is modeled as a collection of agents whose social contacts have a complex network topology. Compared to other modeling approaches of technological progress [1-8], here agents represent individuals that are potential users of newly developed technologies. The behavior of agents is captured by two parameters that characterize their response to the external driving due to advertising activities, and to the word-of-mouth communication with their social partners. Analytic calculations and computer simulations revealed a rich spectrum of novel behaviors varying the parameters of the model and the underlying network topology of agents' social contacts. Agents who got informed through external advertising serve as nucleation centers from which clusters of informed agents grow. The gradual nucleation and growth of clusters lead to merging, which in turn results in the appearance of a macroscopic cluster connecting nearly all informed agents. We show that on the macroscale, the time evolution of the fraction of informed agents is characterized by an integral equation. On the microscale, simulations revealed that at the critical time $t_{c}$ of the emergence of a spanning cluster, the size distribution of clusters becomes a power law. The exponent $\tau$ depends both on the strength of interaction of agents and on the topology of their social contacts: On a square lattice the exponent is $\tau=1 / 2$ when the word-of-mouth communication dominates, while it converges to a higher value $\tau=1.75$ as the external driving gets stronger. Introducing long-range contacts, a crossover occurs to $\tau=2.5$ for large cluster sizes. The critical time $t_{c}$ decreases logarithmically with increasing rewiring probability. 


\section{VERTICAL AND HORIZONTAL INFORMATION CHANNELS}

In our model the socioeconomic system is represented by a set of agents that have a complex topology of social contacts. The state of agents during the time evolution of the system is characterized by an integer variable $S_{i}$, which can have two distinct values

$$
S_{i}= \begin{cases}1, & \text { if agent } i \text { is uninformed } \\ 0, & \text { if agent } i \text { is informed }\end{cases}
$$

When a new innovation is going to be introduced on the market, initially all agents are in the state $S_{i}=1, i=1, \ldots, N_{0}$, where $N_{0}$ denotes the total number of agents.

In the model the primary source of information on the availability of new innovations or products in the market is the advertisement, which is mainly done in the media such as television, radio, the Internet, and newspapers. This information channel is available for all agents, and that is why we assume that it acts as a homogeneous external driving field on the system with field strength $E$. The value of $E$ characterizes the intensity of advertising activities, which could be quantified by the advertising expenditure of the producer. In spite of the homogeneity of the external information field, agents can have different reactions to it, simply due to their different personalities. In order to simplify the problem, we represent the sensitivity of agents to the external information field by a single parameter $0 \leqslant \beta$, which has the same value for all agents.

In a socioeconomic system information can be transmitted between interacting agents by word-of-mouth communication resulting in a horizontal flow of information. The strength of coupling of an agent to its interacting partners would in principle be different for each social contact. For simplicity, we assume that the coupling of agents to their social environment is characterized by a single parameter $0 \leqslant \alpha$, which has the same value for all the connections.

We cast the total amount of information $I_{i}$ agent $i$ receives through the external information field $E$ (vertically) and through its social contacts (horizontally) in the following form:

$$
I_{i}=\alpha S_{i} \sum_{j=1}^{n_{i}}\left(1-S_{j}\right)+\beta S_{i} E,
$$

where $n_{i}$ denotes the number of social contacts of agent $i$. It can be seen that the first term simply counts the number of those social partners of agent $i$ that are already aware of the innovation, since only agents with state variable $S_{j}=0$ contribute to the sum. The second term ensures the coupling of agents to the external information field. Agents that have enough information may become aware of the innovation. The awareness occurs with a certain probability $A$, which is a monotonically increasing function of the available information $I_{i}$ :

$$
A\left(I_{i}\right)=1-e^{-I_{i} / \lambda},
$$

where $\lambda$ is a scale parameter. Note that $I_{i}$ is the total amount of new information received by agent $i$. Hence, when the agent is already aware of the availability of the innovation, its state variable is set to $S_{i}=0$ implying that $I_{i}$ becomes immediately zero. Since the surroundings of a selected agent changes due to the agents that have become aware of the innovation, the total amount of new information $I_{i}$ and the resulting awareness probability $A\left(I_{i}\right)$ are both functions of time.

In this paper we present a detailed study of the model varying the values of $\alpha$ and $\beta$ in a broad range while other parameters of the model are fixed, $E=1, \lambda=1$. In order to control the topology of the social contacts, agents are considered on a square lattice that is then rewired according to the Watts-Strogatz method [28]: Assuming periodic boundary conditions on a square lattice in both directions, each bond of the lattice is removed with a probability $p$, which is then reestablished between two randomly chosen nodes. The rewiring process introduces long-range connections between agents so that varying the value of the rewiring probability $p$, the interaction of agents can be tuned from completely short-range $p=0$, through long range with small-world properties, to Erdös-Rényi graphs, $p=1$ [28-31]. To ensure good statistics of the numerical data, simulations were carried out starting from a square lattice of size $L=2001$, which provides $N_{0} \approx 4 \times 10^{6}$ agents.

Analytic calculations and computer simulations revealed that the competition of the vertical and horizontal information channels controlled by the coupling constants $\alpha$ and $\beta$ results in a complex time evolution of the system. In the following we present the behavior of the system on the macroscale together with the underlying microscopic dynamics and structural features.

\section{MACROSCOPIC TIME EVOLUTION}

The macroscopic time evolution of the system is characterized by the fraction of agents $q(t)=N(t) / N_{0}$, which are aware of the new technology and by the fraction of new adoptions $\delta q(t)$, which is the derivative of the cumulative fraction $\delta q(t)=d q(t) / d t$. Here $N(t)$ denotes the total number of agents already aware of the innovation at time $t$. Figure 1 presents $q(t)$ and $\delta q(t)$ as a function of time $t$ for several values of the rewiring probability $p$ obtained by computer simulations. It can be observed that $q(t)$ is a monotonically increasing function of time for any topologies with a welldefined inflexion point. It is important to emphasize that the overall shape of the adoption curve $q(t)$ always has the typical logistic feature observed for spreading phenomena in socioeconomic systems [1-3]. The fraction of new adoptions $\delta q(t)$ has a maximum whose position $t_{m}$ coincides with the inflexion point of the total fraction of adoptions $q(t)$. It is interesting to note that increasing the rewiring probability $p$, the spreading process gets faster; i.e., the position of the maximum $t_{m}$ of $\delta q(t)$ shifts to the left and $q(t)$ saturates faster. Due to the connectivity of the social network, the total fraction of adoptions $q(t)$ has to converge to 1 with increasing time. (The effect of the network topology on the efficiency of spreading will be quantified in Sec. V together with the effect of the microstructure.) Our results on the effect of the Watts-Strogatz rewiring parability $p$ in the macroscopic time evolution of the system are in qualitative agreement with the generic results of Watts on spreading phenomena occurring on graphs [4]. 


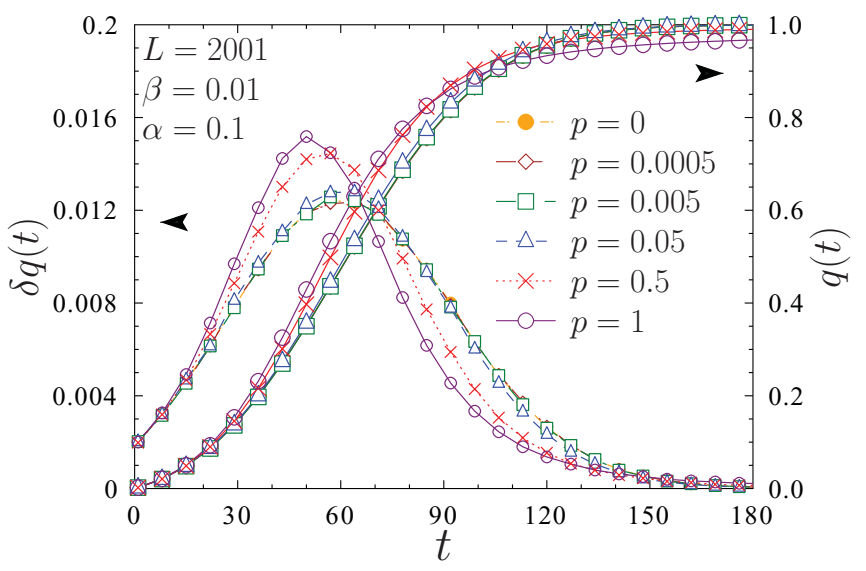

FIG. 1. (Color online) Macroscopic time evolution of the system characterized by the total fraction of agents, $q(t)=N(t) / N_{0}$, which are already aware of the innovation, and by the fraction of new adoption, $\delta q(t)=d q(t) / d t$, for several values of the rewiring probability $p$. The coupling constants are $\alpha=0.1$ and $\beta=0.01$.

In order to obtain an analytical understanding of the macroscopic time evolution we have to investigate the microscopic dynamics of the system. Initially all agents are uninformed about the new technology. As time elapses, a small fraction of agents becomes aware of the innovation due to the external driving. These early adoptions serve as nucleation centers from which clusters of informed agents grow due to the interagent coupling. The finite value of $\delta q$ at $t=0$ in Fig. 1 arises due to these initial nucleations. Since nucleation is an uncorrelated process, growing clusters are spread all over the network homogeneously distributed at random locations. The time evolution of the microstructure of the system is presented in Fig. 2 on a square lattice $(p=0)$. In order to make structural details more visible, we intentionally chose a relatively small system size $L=100$. It can be observed

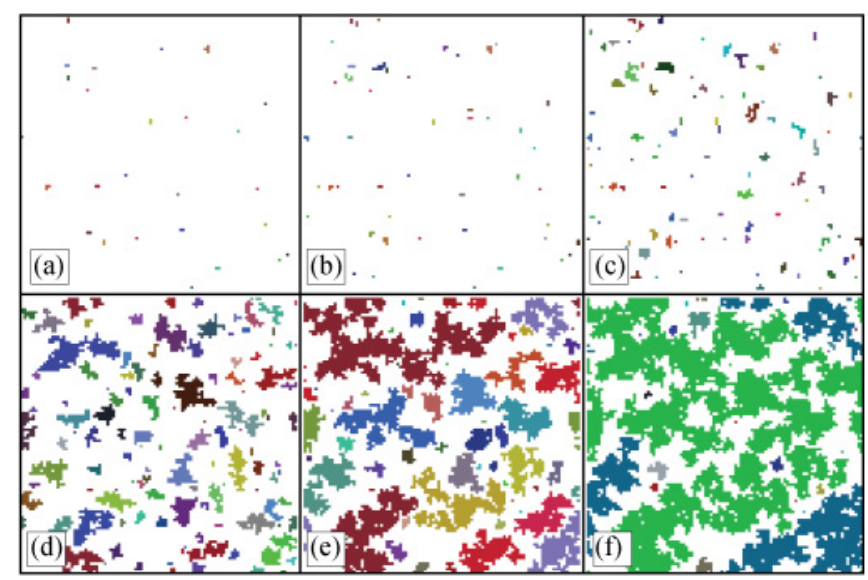

FIG. 2. (Color online) Time evolution of the system on a square lattice $p=0$ of size $L=100$ for the parameter values $\alpha=0.1$ and $\beta=0.01$. It can be observed that clusters nucleate at random positions $(a, b)$, then they grow due to the interagent communication $(c, d, e) .(f)$ A giant cluster can be observed due to the merging of smaller ones. Randomly selected colors are assigned to clusters of different sizes. that at the beginning of the process nucleation of new clusters dominates [Figs. 2(a) and 2(b)]. As time elapses, growing clusters cover an increasing fraction of the lattice so that nucleation slows and lateral spreading through interagent contacts rules the evolution of the system [Figs. 2(c) and 2(d)]. It can be observed that the size of clusters $S$ spreads over a rather broad interval: Due to the new nucleations there are clusters of single agents $S=1$ at any time, while those clusters that nucleated at the beginning of the process could reach rather large sizes due to growing. As clusters grow they can also merge, which results in further increase of the cluster size.

The size $S$ of growing clusters, i.e., the number of informed agents in the cluster, can simply be obtained analytically as a function of time $t$ when merging is neglected. Clusters grow due to the outflow of information through the cluster boundary; hence, the rate of cluster growth is proportional to the cluster perimeter

$$
\frac{d S}{d t} \sim \sqrt{S},
$$

from which the size $S$ of a cluster at time $t$ that was nucleated at time $t^{\prime}<t$ follows as

$$
S\left(t, t^{\prime}\right)=C\left(t-t^{\prime}\right)^{2} .
$$

The multiplication factor $C$ depends on both $\alpha$ and $\beta$. At an arbitrary time new adoptions occur in the system due to two reasons: (1) Nucleation of new informed agents appears with a probability $p_{n}=1-\exp (-\beta E / \lambda)$. (2) Along the boundary of clusters, agents get informed by word-of-mouth communication with the probability $p_{g}=1-\exp (-2 \alpha / \lambda)$. It can be seen in Fig. 2 that the number of informed neighbors of the perimeter sites of clusters depends on the growth stage; i.e., for very small clusters the number of neighbors is one, while for intermediate and large clusters that have a high degree of compactness and a smooth surface, two neighbors are typical. At locations where the cluster surface is highly ramified three informed neighbors can also be present. The statistics of the number of informed neighbors showed that two neighbors are the most typical. This is captured in the expression of $p_{g}$ by the factor $2 \alpha$. Based on the above arguments the fraction of new adoptions $\delta q(t)$ characterizing the macroscopic evolution of the system can be obtained analytically as

$$
\begin{aligned}
\delta q(t)= & {[1-q(t)] p_{n} } \\
& +[1-q(t)] \int_{0}^{t} \frac{d S\left(t, t^{\prime}\right)}{d t}\left[1-q\left(t^{\prime}\right)\right] p_{n} d t^{\prime} .
\end{aligned}
$$

The first term of Eq. (6) is the fraction of new nucleations at time $t$ taking into account the decreasing fraction of uninformed agents $1-q(t)$. The second term describes the growth of clusters, where the integration is carried out over the nucleation time $t^{\prime}$ of the clusters' seeds. The expression of $S\left(t, t^{\prime}\right)$ has to be substituted from Eq. (5). Since $\delta q(t)$ depends on the present and past values of $q(t)$, Eq. (6) can be solved only numerically. It can be expected that Eq. (6) provides a good description of the macroscopic time evolution of the system if cluster growth dominates over nucleation $p_{n} \ll p_{g}$. Figure 3 presents that for the parameter values $\alpha=10$ and $\beta=0.01$ the numerical solution of Eq. (6) has a very good agreement with the results of computer simulations. Stronger deviations from 


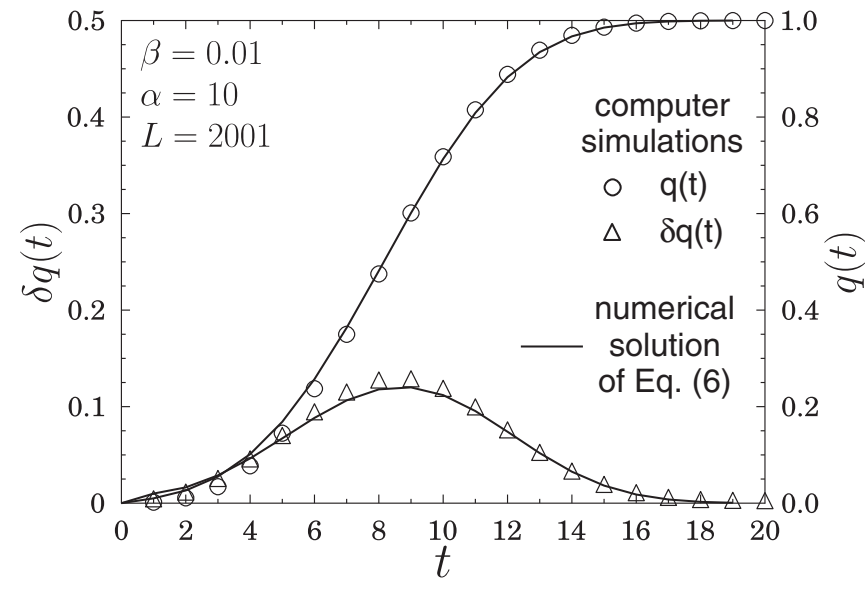

FIG. 3. Comparison of the numerical solution of the analytic expression Eq. (6) and of the results of computer simulations for $q(t)$ and $\delta q(t)$. High-quality agreement is obtained for both quantities with the parameter values $\alpha=10, \beta=0.01, L=2001$, and $p=0$.

the analytical description with Eq. (6) are usually observed for the parameter regime $p_{g}<p_{n}$.

\section{MICROSTRUCTURE OF THE SYSTEM}

It can be observed in Fig. 2 that at a given time $t$ clusters of widely different sizes are present in the system: The smallest one has size $S=1$ just nucleated due to the external driving, while, neglecting the merging of clusters, the largest one has a size $S_{\max }=C t^{2}$ because it was nucleated right in the initial state. At low enough nucleation rate $p_{n} \ll p_{g}$ the density of clusters on the social network remains low over considerable times, allowing for the dominance of lateral spreading. Under such conditions the variations of cluster sizes are solely caused by the different nucleation time $t^{\prime}$ of the clusters' seeds in the expression Eq. (6) of $S\left(t, t^{\prime}\right)$. (We note that for the parameter setting $E=1, \lambda=1$ used in our study the nucleation probability can be estimated as $p_{n} \approx \beta$, for $\beta \ll 1$.)

\section{A. Cluster size distribution}

The number of clusters $d N$ nucleated in an infinitezimal time interval $d t^{\prime}$ at time $t^{\prime}$ can be obtained as $d N=\left[N_{0}-\right.$ $\left.N\left(t^{\prime}\right)\right] p_{n} d t^{\prime}$. Neglecting the presence of already informed agents $N(t) \ll N_{0}$ and taking into account the growth law Eq. (5) of clusters, the size distribution of clusters $P_{t}(S)$ at time $t$ can be obtained analytically as

$$
P_{t}(S) \simeq \frac{p_{n} N_{0}}{2 C^{1 / 2} S_{\max }^{1 / 2}} S^{-1 / 2},
$$

where $t$ dependence occurs only in the upper limit of cluster sizes $1<S<S_{\max }(t)$. Figure 4(a) presents the cluster size distribution at different times in an evolving system. Note that the parameters $\alpha=10^{-1}$ and $\beta=10^{-7}$ are intentionally chosen such that growth dominates over a considerable time without too many merging events. Good agreement is obtained with the analytical prediction verifying the validity of our assumptions. As time elapses, deviations from Eq. (7) occur due to two mechanisms that are not captured by the above analysis: (1) The increasing number of informed agents
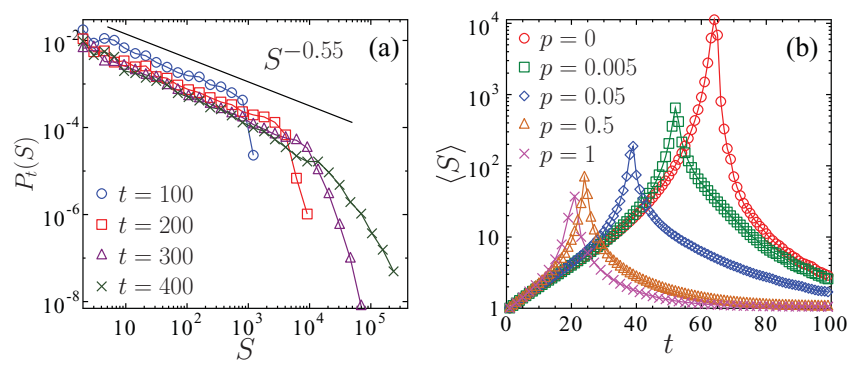

FIG. 4. (Color online) (a) Size distribution of clusters on a square lattice of size $L=2001$ for the parameters $\alpha=10^{-1}$ and $\beta=10^{-7}$. At early times a good agreement can be observed with the analytical prediction Eq. (7); however, as time elapses the deviations become more and more apparent. (b) Average cluster size $\langle S\rangle$ as a function of time for several values of the rewiring probability $p$ for $\alpha=0.1$ and $\beta=0.01$. A sharp maximum appears that indicates the formation of a dominating cluster of informed agents.

reduces the available space for the new nucleations, which suppresses the fraction of small clusters. (2) Merging of growing clusters increases the cluster size and decreases their frequency of appearance. Computer simulations revealed that as time elapses the cluster size distributions undergo an evolution where the exponent of the power-law regime tends to a limit value.

In order to characterize the growth of clusters and the evolution of their size distribution, we determined the average cluster size $\langle S\rangle$ as a function of time $t .\langle S\rangle$ is defined as the ratio of the second and first moments of the cluster size distribution

$$
\langle S\rangle(t)=\frac{\sum_{i} S_{i}^{2}}{\sum_{i} S_{i}},
$$

where $S_{i}$ denotes the size of cluster $i$ in a snapshot at time $t$. In the evaluation the largest cluster is always excluded. It can be seen in Fig. 4(b) that $\langle S\rangle(t)$ has a sharp maximum, which indicates the emergence of a giant cluster on the complex network of social contacts as a consequence of merging of growing clusters. The position of the maximum determines the critical point $t_{c}$ of the system. The presence of a single dominating cluster means that for $t>t_{c}$ a macroscopic fraction of agents is already aware of the innovation. It is interesting to note that the qualitative shape of the $\langle S\rangle(t)$ curve is the same for all rewiring probabilities; however, with increasing $p$ the growth process gets faster, indicated by the decreasing value of $t_{c}$.

As to the next we evaluated the size distribution of cluster $P_{t}(S)$ at different times $t$ approaching $t_{c}$. Figure 5(a) presents cluster size distributions on a square lattice of agents $p=0$ at different time values $t$ up to the critical time $t_{c}$ for the parameters $\alpha=0.1$ and $\beta=0.01$. At these values of the coupling constants the initial power-law regime with exponent $1 / 2$ is hardly visible (compare Fig. 4), as time elapses the distribution becomes a steeper power law followed by an exponential cutoff. It can be observed in Fig. 5(a) that approaching the critical point $t_{c}$, the distribution function spans a broader range of cluster sizes, and finally at $t=t_{c}$ it becomes a high-quality power law

$$
P_{t}(S) \sim S^{-\tau},
$$



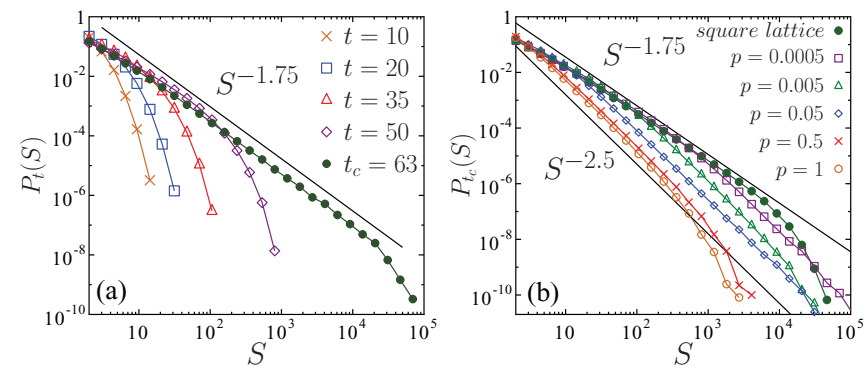

FIG. 5. (Color online) Size distribution of clusters on a square lattice (a) and on complex networks (b) for the parameters $\alpha=0.1$ and $\beta=0.01$. Approaching $t_{c}$ on a square lattice in (a) a powerlaw form is obtained with the exponent $\tau=1.75$. When long-range connections are present, for large cluster sizes a crossover is obtained to a higher exponent $\tau=2.5$.

where the value of the exponent was obtained numerically as $\tau=1.75 \pm 0.05$.

It is an important question how the network topology influences the functional form of the cluster size distributions $P_{t}(S)$ and the value of the exponent $\tau$. Figure 5(b) presents $P_{t_{c}}(S)$ determined at the critical point $t=t_{c}$ varying the topology of the network of agents with the rewiring probability. It can be observed in Figure 5(b) that the presence of long-range social contact primarily affects the regime of large clusters of the size distribution. As the rewiring probability $p$ is increased from zero, for small clusters the exponent obtained on the regular square lattice prevails $\tau=1.75 \pm 0.05$; however, for large cluster sizes a crossover occurs to a higher exponent, $\tau=2.5 \pm 0.07$. The crossover cluster size separating the regimes of different exponents is a decreasing function of the rewiring probability $p$. It is important to note that the cluster size exponent $\tau$ of the spreading process obtained on the square lattice is considerably lower than the one of percolation in two dimensions, $\tau_{p} \approx 2.05$ [32]. However, when long-range connections appear in the system, the value of $\tau$ coincides with that of site percolation on small-world and random graph topologies, $\tau_{p}=5 / 2$ [29-31].

\section{B. Geometrical structure of clusters}

It can be observed in Fig. 2 that on the square lattice small clusters are compact while the large ones are very disordered having holes inside and an irregular surface. In order to quantify the geometrical structure of clusters on a square lattice, we calculated the radius of gyration $R_{g}$ of clusters with the definition

$$
R_{g}^{2}=\frac{1}{S(S-1)} \sum_{i \neq j=1}^{S}\left(\vec{r}_{i}-\vec{r}_{j}\right)^{2},
$$

where $\vec{r}_{i}$ denotes the position of agent $i$ inside the cluster. In Fig. 6 the size of clusters $S$ is presented as a function of the radius of gyration $R_{g}$. It is interesting to note that on a double logarithmic plot the functional form of $S\left(R_{g}\right)$ is composed of two power-law regimes with different exponents

$$
S \sim R_{g}^{D}
$$

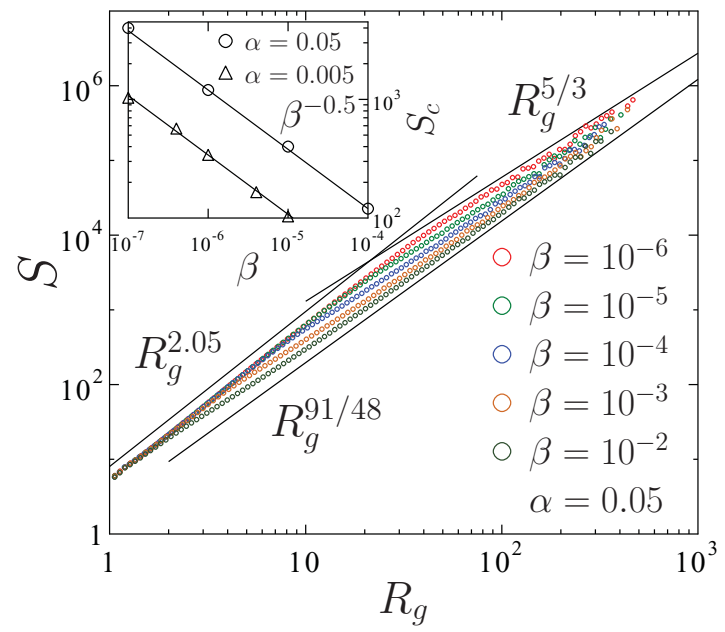

FIG. 6. (Color online) The size of clusters $S$ as a function of the linear extension $R_{g}$. Power-law behavior is evidenced with a crossover from a compact space-filling form to a fractal structure with increasing cluster size. The value of $\beta$ increases from top to bottom in the range of large $\beta$ values. Inset: The crossover cluster size $S_{c}$ has a power-law dependence on the value of $\beta$.

Since small clusters are compact, they are described by $D=2$; however, due to the ramified structure of large clusters a crossover occurs to a significantly lower fractal dimension $D \approx 5 / 3$. The crossover between the compact and fractal cluster structures occurs at a characteristic cluster size $S_{c}$, which solely depends on the nucleation rate $\beta$. At low nucleation rate $\beta \ll \alpha$ clusters can grow large before merging; hence, the crossover can be observed only for the largest clusters. It can be seen in Fig. 6 that increasing $\beta$ the crossover cluster size $S_{c}$ decreases while the fractal dimension $D$ gets larger. The reason is that at higher values of $\beta$ nucleation dominates the time evolution of the system; i.e., within a few time steps the random nucleation leads to percolation, leaving hardly any role for the growth of clusters. Consequently, $S_{c}$ decreases while the value of $D$ increases to the fractal dimension of percolation clusters, $D=91 / 48$ (see Fig. 6) [32].

The inset of Fig. 6 presents the crossover cluster size $S_{c}$ as a function of $\beta$. A power-law dependence is obtained:

$$
S_{c} \sim \beta^{-\kappa},
$$

where the exponent $\kappa=1 / 2$ proved to be independent of the value of interagent coupling $\alpha$. The crossover from compact to fractal clusters marks the point where merging of clusters starts dominating, which affects also the global time evolution of the system: Merging has the consequence that the total cluster surface starts to decrease, which implies the slowing down of new adoptions $\delta q(t)$ and leading finally to the maximum of $\delta q(t)$.

\section{THE EFFECT OF CLUSTER STRUCTURE ON THE TIME SCALE OF SPREADING}

When the coupling to the external field has a low strength $p_{n} \ll p_{g}$, the information reaches most of the agents through their social contacts. In this case the cluster structure of 


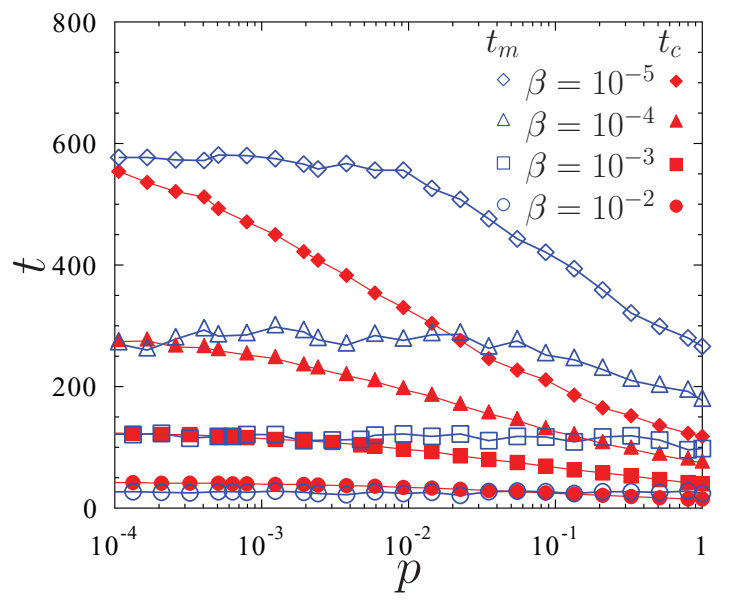

FIG. 7. (Color online) The critical time $t_{c}$ and the position $t_{m}$ of the maximum of the curve of new adoptions $\delta q(t)$ as a function of the rewiring probability $p$ for several $\beta$ values with fixed $\alpha=0.01$. As $\beta$ increases, $t_{m}$ becomes independent of $p$ while $t_{c}$ decreases logarithmically.

informed agents may have a strong effect on the efficiency of information transfer to the uninformed agents. The question naturally arises how the appearance of the dominating cluster at the critical point $t_{c}$ affects the spreading process. To obtain a quantitative answer, in Fig. 7 we show the value of the critical time $t_{c}$, i.e., the position of the maximum of the average cluster size $\langle S\rangle$, and the position $t_{m}$ of the maximum of the new adoptions $\delta q(t)$ as a function of the rewiring probability for several values of $\beta$ in the range $\beta \leqslant \alpha$. It can be seen in the figure that on the square lattice $(p=0)$ the values of $t_{m}$ and $t_{c}$ always coincide. The reason is that the main source of spreading is the interagent communication, which mainly occurs along the boundaries of the clusters. Hence, when the giant cluster appears, the free cluster surface drops down, which results in a decrease of $\delta q(t)$. At finite rewiring probabilities $0<p$, however, the giant cluster appears before $\delta q(t)$ reaches its maximum; more specifically, with increasing $p$ the value of the critical time $t_{c}$ decreases, while the value of $t_{m}$ hardly changes. Increasing the nucleation probability $\beta$ the value of $t_{m}$ becomes more and more independent of $p$. When long-range connections are present in the system, the appearance of a dominating cluster does not imply the decrease of the fraction of new adoptions. On the contrary, the spreading efficiency characterized by $\delta q(t)$ can still increase. Note that the nearly linear behavior of $t_{c}$ on the semilogarithmic plot of Fig. 7 implies the logarithmic dependence

$$
t_{c} \sim \ln p^{-1}
$$

on the rewiring probability.

\section{DISCUSSION}

We investigated the competition of external driving and of word-of-mouth communication in a social system where information is spreading about some products newly introduced in the market. The external source of information is the producers' advertising activity, which induces the initial market penetration through the most sensitive agents. Parallel to the external driving, information spreads laterally through the social contacts of individuals. Compared to models of social percolation, in our system the effect of the external field is not restricted to the beginning of the information transfer; instead it acts continuously during the entire time evolution of the system. Furthermore, we do not consider the opinion of agents about the product, just the fact that they are aware of its advantages. Varying the relative importance of the vertical and horizontal information channels, we studied both the macroscopic time evolution and the microstructure of the system. On the macrolevel the system is characterized by the fraction of informed agents and by the fraction of new adoptions. Informed agents form clusters that grow and merge, covering eventually the entire social network.

Computer simulations showed that the total fraction of informed agents has the logistic shape usually observed in the diffusion of innovations [2]. Based on the microscopic mechanism of nucleation and growth of clusters, the macroscopic time evolution can be described by an integral equation, which provides satisfactory results when growth dominates the spreading process.

The microstructure of the system characterized by the size distribution of clusters and by the geometrical structure of individual clusters strongly depends on the competition of the two information channels, which can be characterized by the ratio of the probabilities of nucleation and cluster growth. In order to provide a clear overview of the structure and dynamics we constructed an approximate phase diagram of a square lattice of agents on the $\alpha-\beta$ plane, which is presented in Fig. 8. When the growth of clusters dominates over nucleation, i.e., below the dashed line of $p_{n} / p_{g} \approx$ $5 \times 10^{-5}$, the size distribution of clusters has a power-law behavior with an exponent $\tau=1 / 2$. The clusters are compact characterized by $D=2$ with a crossover to a lower fractal dimension $D=5 / 3$ when merging starts. When nucleation,

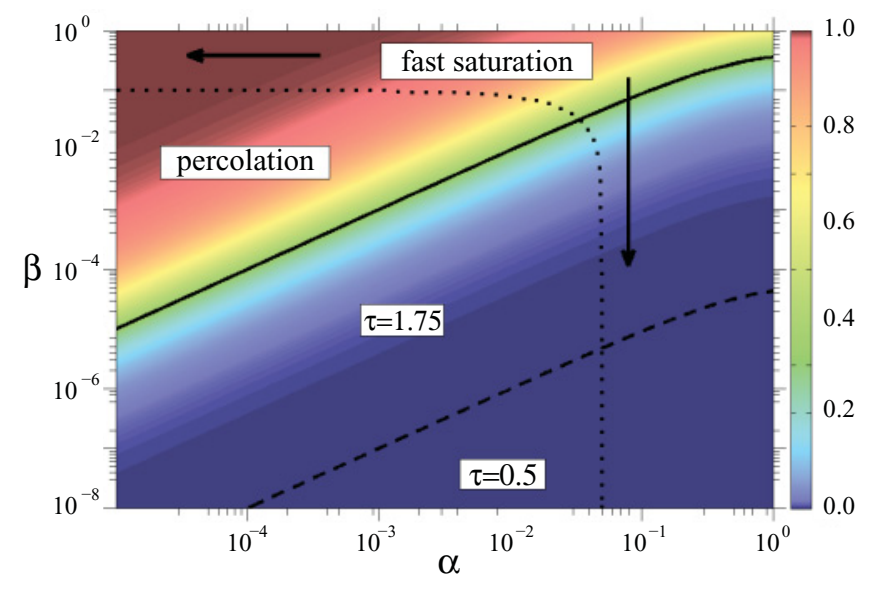

FIG. 8. (Color online) Phase diagram of the system on the $\alpha-\beta$ plane. The color code represents the value of the ratio of the probabilities of nucleation and growth $p_{n} / p_{g}$. The dashed and continuous lines indicate the parameters satisfying the conditions $p_{n} / p_{g}=5 \times 10^{-5}$ and $p_{n} / p_{g}=0.1$, respectively. In the percolation phase the critical state of the system where the spanning cluster is formed is practically identical with that of percolation in two dimensions on a square lattice. Outside the line of fast saturation the spanning cluster appears already in the first time step. 
growth, and merging have a more-or-less balanced role, i.e., between the continuous line of $p_{n} / p_{g} \approx 0.1$ and the dashed line of $p_{n} / p_{g} \approx 5 \times 10^{-5}$, the size distribution remains a power law; however, the exponent $\tau$ becomes significantly higher, $\tau \approx 1.75$. Simulations revealed that the topology of the social contacts of agents has a very strong effect on the cluster structure: Increasing the fraction of long-range contacts a crossover occurs between two power-law regimes; i.e., for small cluster sizes the exponent characterizing regular lattices prevails, $\tau \approx 1.75$; however, for large clusters a second power-law regime emerges with a higher exponent, $\tau \approx 2.5$. When nucleation dominates the time evolution, the fraction of informed agents reaches the percolation threshold within a few time steps, and the behavior of the system becomes similar to the one of percolation lattices. Outside the curve of fast saturation on the parameter plane of Fig. 8 the percolation threshold is passed already in the second iteration step.

We demonstrated that the cluster structure of the system has an effect also on the macroscopic time evolution and on the speed of adoptions: When word-of-mouth communication dominates, the merging of clusters reduces the free cluster surface where communication mainly occurs. It has the consequence that on regular lattices whenever a giant cluster appears, the spreading process slows. It implies that a stronger advertising activity is required from the producer of the technology in order to keep the spreading process advancing
$[1,2]$. However, on a realistic complex network of individuals the formation of a macroscopic connected cluster of informed agents does not imply the drop down of spreading. Realistic complex networks of individuals are more efficient because long-range connections contribute to nucleation, reducing the need for extra advertising efforts (as seen in Sec. V). The critical time $t_{c}$ when the macroscopic cluster appears has a logarithmic dependence on the rewiring probability.

Our study was restricted to the information spreading without considering the purchase of the new product. An interesting next step of the investigations is to complement the model with a stochastic rule to capture the typical customer behavior of informed agents, allowing also for the variation of the coupling constants $\alpha$ and $\beta$.

\section{ACKNOWLEDGMENTS}

The work is supported by the TAMOP-4.2.1/B-09/1/ KONV-2010-0007 project. The project is implemented through the New Hungary Development Plan, cofinanced by the European Social Fund and the European Regional Development Fund. FK acknowledges support of the Janos Bolyai project of HAS and of OTKA K84157. This work was carried out with the support of Toyota Central R\&D Labs, Aichi, Japan.
[1] E. M. Rogers, Diffusion of Innovations, 5th ed. (New York, Free Press, 2003).

[2] V. Mahajan and R. A. Peterson, Models for Innovation Diffusion, Sage University Paper Series on Quantitative Applications in the Social Sciences, Vol. 48 (Sage, Newbury Park, CA, 1985).

[3] W. Weidlich, Sociodynamics: A Systematic Approach to Mathematical Modeling in the Social Sciences (Dover Publications, Mineola, NY, 2000).

[4] D. J. Watts, Am. J. Sociol. 105, 493 (1999).

[5] R. Andergassen, F. Nardini, and M. Ricottili, J. Econ. Dyn. Control 33, 1779 (2009).

[6] R. Andergassen, F. Nardini, and M. Ricottili, J. Econ. Behav. Organ. 61, 710 (2006).

[7] A. Arenas, A. Díaz-Guilera, C. J. Pérez, and F. Vega-Redondo, J. Econ. Dyn. Control 26, 2115 (2002).

[8] S. Valverde, R. V. Solé, M. A. Bedau, and N. Packard, Phys. Rev. E 76, 056118 (2007).

[9] F. Kun, G. Kocsis, and J. Farkas, Physica A 383, 660 (2007).

[10] G. Kocsis and F. Kun, J. Stat. Mech. (2008) P10014.

[11] R. M. Ruiz, E. Albuquerque, L. C. Ribeiro, and A. T. Bernardes, AIP Conf. Proc. 779, 162 (2005).

[12] D. Helbing, M. Treiber, and N. J. Saam, Phys. Rev. E 71, 067101 (2005).

[13] C. Castellano, S. Fortunato, and V. Loreto, Rev. Mod. Phys. 81, 591 (2009).

[14] S. Solomon, G. Weisbuch, L. de Arcangelis, N. Jan, and D. Stauffer, Physica A 277, 239 (2000).
[15] A. Proykova and D. Stauffer, Physica A 312, 300 (2002).

[16] K. Sznajd-Weron and J. Sznajd, Int. J. Mod. Phys. C 11, 1157 (2000).

[17] K. Sznajd-Weron and R. Weron, Int. J. Mod. Phys. C 13, 115 (2002).

[18] W. Duan, Z. Chen, Z. Liu, and W. Jin, Phys. Rev. E 72, 026133 (2005).

[19] A. Arenas, A. Díaz-Guilera, C. J. Pérez, and F. Vega-Redondo, Phys. Rev. E 61, 3466 (2000).

[20] X. Guardiola, A. Diaz-Guilera, C. J. Pérez, A. Arenas, and M. Llas, Phys. Rev. E 66, 026121 (2002).

[21] H.-B. Hu and X.-F. Wang, J. Phys. A 42, 225005 (2009).

[22] D. Stauffer, JASSS 5, no. 1, paper 4.

[23] D. Stauffer, Int. J. Mod. Phys. C 13, 315 (2002).

[24] A. T. Bernardes, D. Stauffer, and J. Kertész, Eur. Phys. J. B 25, 123 (2002).

[25] K. Sznajd-Weron, Acta Phys. Pol. B 36, 2537 (2005).

[26] R. Hegselmann and U. Krause, JASSS 5, no. 3, paper 2.

[27] G. Silverberg and B. Verspagen, J. Econ. Dyn. Control 29, 225 (2005).

[28] D. J. Watts and S. H. Strogatz, Nature (London) 393, 440 (1998).

[29] R. Albert and A.-L. Barabási, Rev. Mod. Phys. 74, 47 (2002).

[30] M. E. J. Newmann, SIAM Rev. 45, 167 (2003).

[31] R. Pastor-Satorras, M. Rubi, and A. Diaz-Guilera, Statistical Mechanics of Complex Networks, Lecture Notes in Physics, Vol. 625 (Springer Verlag, Berlin, 2003).

[32] D. Stauffer and A. Aharoni, Introduction to Percolation Theory (Taylor and Francis, New York, 1994). 